九州大学学術情報リポジトリ

Kyushu University Institutional Repository

\title{
Acceptability of a Decision Maker to Handle Multi-objective Optimization on Design Space
}

Inoue, Makoto

Dept. of Civil Engineering and Architecture, National Institute of Technology, Akita College

高木，英行

Faculty of Design, Kyushu University

http://hdl. handle. net/2324/4488109

出版情報: 2020 Joint 11 th International Conference on Soft Computing and Intelligent Systems and 21 st International Symposium on Advanced Intelligent Systems (SCIS-ISIS), pp.55-63, 202012-05. IEEE

バージョン :

権利関係 : $\odot 2006$ IEEE. Personal use of this material is permitted. Permission from IEEE must be obtained for all other uses, in any current or future media, including reprinting/republishing this material for advertising or promotional purposes, creating new collective works, for resale or redistribution to servers or lists, or reuse of any copyrighted component of this work in other works. 


\section{Acceptability of a Decision Maker to Handle Multi-objective Optimization on Design Space}

\author{
$1^{\text {st }}$ Makoto Inoue \\ Dept. of Civil Engineering and Architecture \\ National Institute of Technology, Akita College \\ Akita, Japan \\ ORCID 0000-0002-9324-2628
}

\author{
$2^{\text {nd }}$ Hibiki Matsumoto \\ Osaka Division \\ TAK-QS Co., Ltd. \\ Osaka, Japan
}

\author{
$3^{\text {rd }}$ Hideyuki Takagi \\ Faculty of Design \\ Kyushu University \\ Fukuoka, Japan \\ ORCID 0000-0002-1431-9472
}

\begin{abstract}
We introduce the acceptability of a decision maker to handle evolutionary multi-objective optimization (EMO) on design space, while most of EMO research tries to find many solutions on an objective space and passes them to a decision maker. Unlike this conventional EMO approaches, our approach decides maker's model with the concept of acceptability and introduces it in EMO search. Especially, this approach works well when qualitative factors, such as the decision maker's experience and knowledge on a task, are a part of evaluations. Acceptability functions for each of objectives are aggregated firstly, and the aggregated acceptability forms contours on an objective space and is mapped on a design space. The acceptability contours on a design space can narrow down the area of solutions. We could find better solutions in our experiments than the conventional approach of searching solutions on an objective space.

Index Terms-qualitative factors, decision making, manyobjective problem, interactive system, hybrid rocket engine
\end{abstract}

\section{INTRODUCTION}

Many practical optimization tasks in the real-world have multiple objectives. Almost all current evolutionary multiobjective optimization (EMO) [2] take the Pareto approach [6] to optimize such tasks. Their goal is to find non-dominated solutions as much as possible. These many solutions are sent to a decision maker to select the final practical solution(s). The biggest feature of this current EMO approach is to separate the stage of finding candidate solutions and that of deciding the final practical solution(s) completely. In other word, EMO does not care of a decision maker's experiences and knowledge on deciding the final solution during its optimization search.

However, this approach has several limitations. Firstly, since a decision maker has experiences and knowledge to decide the final practical solution, EMO search without them have to be less efficient.

Secondly, it makes a decision maker difficult to find the final practical solution because of quite large number of EMO candidate solutions. It is time consuming for EMO to search candidate solutions that are no scope for decision maker's consideration.

Thirdly, the number of Pareto solutions increase exponentially according to the number of objectives. It results high computational cost in EMO search, and too many candidate solutions are meaningless for a decision maker to find one or two practical solutions.
Fourthly, EMO algorithms do not or cannot embed specific knowledge of the current task because they are designed for generic multi-objective tasks. Since a decision maker focuses only one specific task, he or she tries to find the final practical solution using his/her knowledge on the specific task. However, EMO algorithms are too generic to use the specific domain knowledge and have to search solutions based on a quite qualitative policy, i.e. "the smaller or bigger, the better", which results finding too many Pareto solutions and expensive computational cost.

Finally, current EMO approach has a gap between design space for searching candidate solutions and an objective space for evaluating them. Single objective optimization search candidate solutions and evaluate them on the same design space. So far, good bridges between these two spaces have not proposed.

The objective of this paper is to introduce the concept of acceptability, which is the acceptance degree for each objective based on the experience and knowledge of decision makers, into the stage of finding candidate solutions to overcome these remained problems and evaluate its effect.

We use the conceptual design evaluation tool [16] opened to the public by Japan Aerospace Exploration Agency, JAXA, for a task of hybrid rocket engine [1], [14]. We conduct experiments on multi-objective optimization using acceptance.

\section{RELATED WORKS}

\section{A. Prior Research}

One of major problems of EMO is computational cost when becomes computationally expensive the number of objectives is large [8], [11] Its main reason is due to Pareto approach [6] of EMO, such as NSGA-II [3].

MOEA/D [18] with distributed search vectors and NSGA-III [5] with reference lines are some of them. Their search directions can be restricted thanks to the given search vectors and reference lines. EMO methods using these approaches have been proposed, for example, reference point based NSGAII [4], interactive MOEA/D [7], EMO taking account of the direction of user's preference with reference line [13], and others. 


\section{B. Acceptability}

We define the concept of acceptability as the degree of acceptance or desirability based on the decision maker's preferences, experience, and knowledge for each objective of solutions. Unlike monotonic qualitative search policy of "the smaller or bigger, the better", a decision maker can design

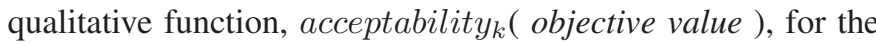
$k$-th objective. It is no need to be a monotonic function but can be any shape based on the decision maker's knowledge on each objective. Furthermore, a decision maker has knowledge of importance degrees among objectives, and they can be expressed as the amplitude of each acceptability function and used for EMO search.

We define one aggregated acceptability function by aggregating acceptability functions using additive, multiplicative or other arithmetic operations on an objective space. Its value becomes a single index or evaluation value of EMO search, is the reflection of decision maker's preference or knowledge of a specific task, and can be used to narrow search areas. Since each solution has one aggregated acceptability value, these values are described on both an objective space and a design space. This is, the aggregated acceptability function can be a bridge connecting both space, which must be help to further develop EMO research.

Note that it is not a simple classical weighting method that approximates a multi-objective optimization to a singleobjective optimization as $\sum w_{i} * o b j_{i}$, where $o b j_{i}$ is the normalized value of the the $i$-th objective and $w_{i}$ is a weight for the value. On the other hand, aggregated acceptability

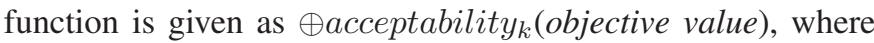
$\oplus$ is an aggregation operator such as additive, multiplicative or other arithmetic operator. This aggregation method has similarities with utility functions [12].

We proposed the concept of this acceptability and confirmed its effectiveness through several experiments. They included an artificial toy task [17], apartment search from rental housing database [9], [15], and silent Silent SuperSonic Technology Demonstrator (S3TD) [10], and we confirmed that introducing domain knowledge of a decision maker is quite useful for multi-objective optimization search.

\section{EXPERIMENTS}

\section{A. Task}

Experimental task is designing a hybrid rocket engine (Fig. 1) with six design variables: initial radius of port [m], fuel length $[\mathrm{m}]$, pressure of combustion chamber [BAR], combustion time [sec], initial mass flow of oxidizer [ $[\mathrm{kg} / \mathrm{sec}]$, and aperture ratio of nozzle [-]. Each variable has five discrete values; the maximum number of possible solutions is 15,625 $\left(=5^{6}\right)$. Total thrust loss coefficient, $\eta_{T}$, is set to 1.0. The range of the initial design variables is as shown in Fig. 4 for the zeroth time.

Two objectives of optimization are gross weight [kg], $W_{\text {gross }}$, (minimum) and flight altitude $[\mathrm{km}], H_{\max }$ (maximum). Conceptual design evaluation tool inputs design variables and outputs these objective values.

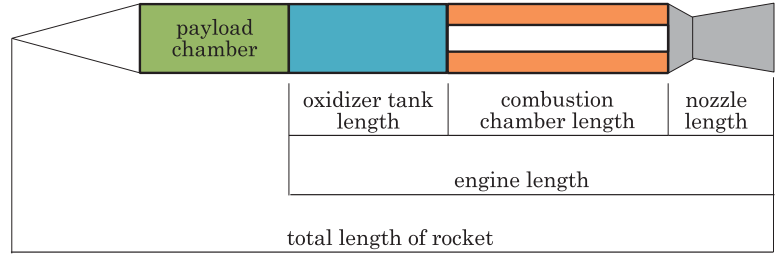

Fig. 1. Outline of a hybrid rocket.

\section{B. Acceptability}

We interviewed two hybrid rocket engine researchers and designed acceptability functions for $W_{\text {gross }}$ and $H_{\max }$ based on their design experiences as shown in Fig. 2; they said "I want $W_{\text {gross }}$ to be in the range of $200-500 \mathrm{~kg}$ (with a lower limit of 150) and $H_{\max }$ to be in the range of $80-160 \mathrm{~km}$ (with an upper limit of 180)" and "I want $W_{\text {gross }}$ to be in the range of $200-300 \mathrm{~kg}$ and $H_{\max }$ to be in the range of $100-160 \mathrm{~km}$ (with an upper limit of 180)." When a project team creates an actual or prototype machine, it is necessary to obtain a few solutions. In such cases, a leader will make decisions as a team representative. Determining the acceptability functions is such an act.
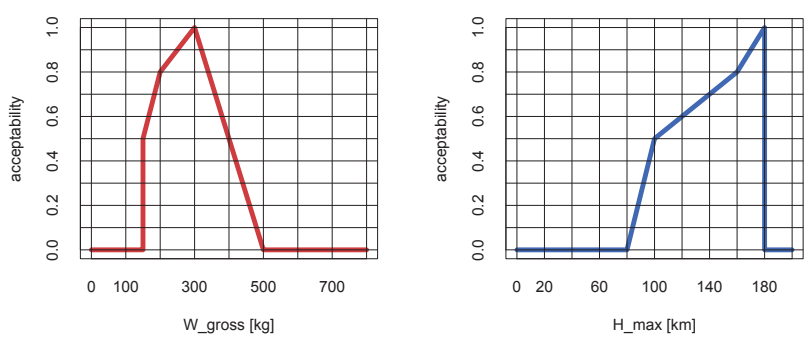

Fig. 2. Acceptability functions. (left) $W_{\text {gross }}$, (right) $H_{\max }$.

The acceptability values are obtained by inputting objective values to these acceptability functions. Aggregated acceptability function over whole objective space is obtained by synthesizing these two acceptability functions; concretely speaking, we use an addition operator for this synthesis in this paper. This is, an aggregated acceptability value is obtained by adding acceptability values for $W_{\text {gross }}$ and $H_{\max }$, and it corresponds to a fitness value of single objective optimization.

\section{Experimental method and flow}

We tackle multi-objective optimization using the concept of acceptability. We set up design variables, compute objective values using a conceptual design evaluation tool provided by JAXA, input the objective values into the acceptability functions of Fig. 2, and compute acceptability values. We create a contour graph of the acceptability on the design space and consider the range of design variables for the next calculation. This process is repeated for optimization. 
Fig. 3 show the flow of our experiment consisting of setting the design variables, calculating the objective value, calculating the acceptability and aggregated acceptability, drawing contour lines, and examining the range of design variables for the next calculation with contour lines. This loop is repeated until it is optimized. This time, we repeat it five times.

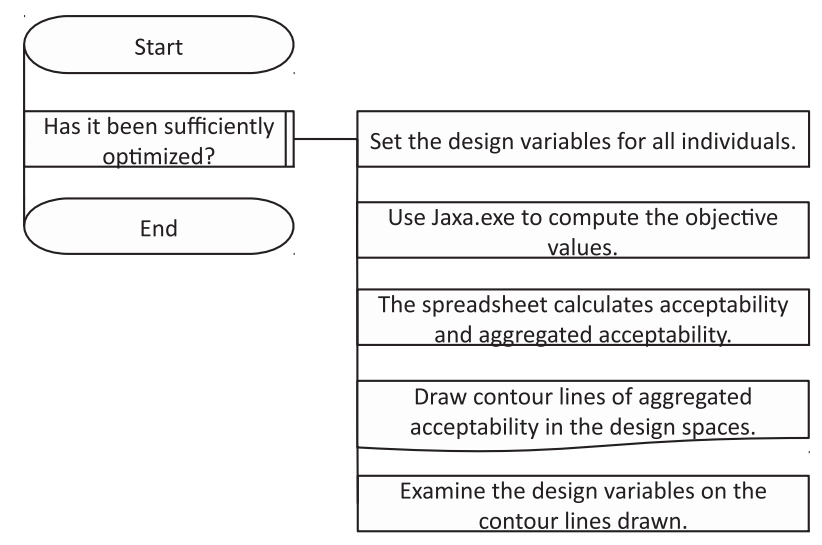

Fig. 3. Problem Analysis Diagram of the experimental flow.

We draw contours on each 2-D space among a 6-D design variable space (total $15={ }_{6} \mathrm{C}_{2}$ ) using LOESS (locally estimated scatterplot smoothing) in an $\mathrm{R}$ package (Fig.s 5 - 13). The interval between contour lines is set to 0.005 aggregated acceptability. Black dots and red ones in the figures indicate the positions of all individuals and the individual with the highest aggregated acceptability, respectively.

Designers look at the drawn contour lines and estimate the promising range of design variables for the next calculation by paying attentions to the locations of the highest contour peaks and the individuals with the highest aggregated acceptability. For instance, the peak of the contour lines in the Fig. 6 locates in $[5,15]$ of initial mass flow of oxidizer, and its maximum value is about 7.5. However, its contour peaks in other figures locate in $[20,25]$. Thus, the range of initial mass flow of oxidizer for the next calculation should be in $[5,25]$. Better values of other design variables are estimated in the same way, too.

\section{RESULTS}

Thanks to the counter curves in a design parameter space and designers' visual estimation, the ranges of design variables become narrower according to calculations (Fig. 4). Fig.s 5-9 show 5 among 15 aggregated acceptability contours for initial mass flow of oxidizer and other combinations after the first calculation.

Fig. 10 - 12 shows how this approach narrows search area in a design parameter space according to search time. They are aggregated acceptability contours at the initial setting, the first calculation, the second calculation, the third calculation, and the fourth calculation, respectively. The calculation time was a little over 30 seconds per an individual with Intel Core i7-3770, and it took about 6 days to calculate all 15,625 individuals at one time.
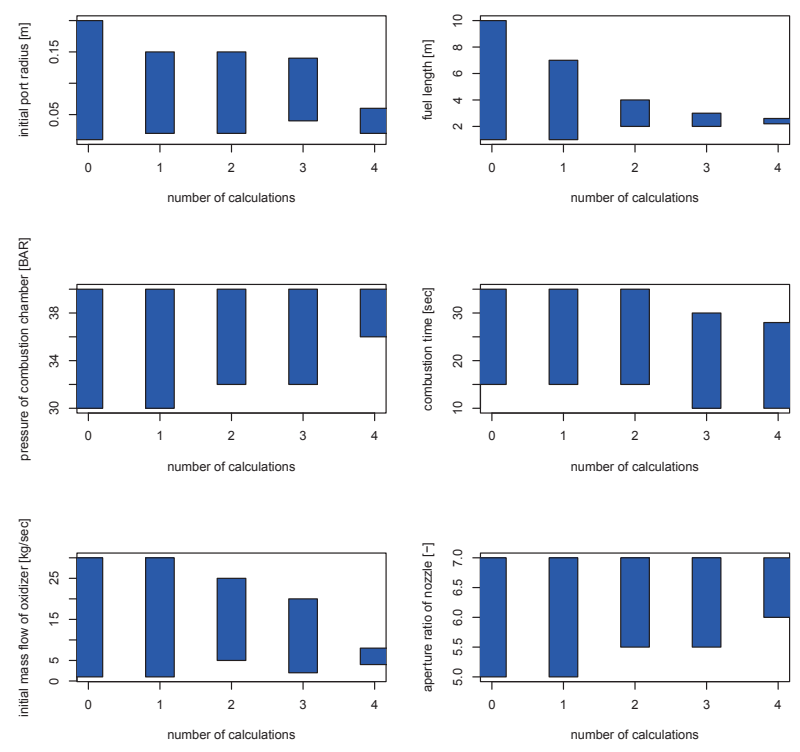

Fig. 4. Visually estimated range of design variables per calculations. Zero means the initial settings

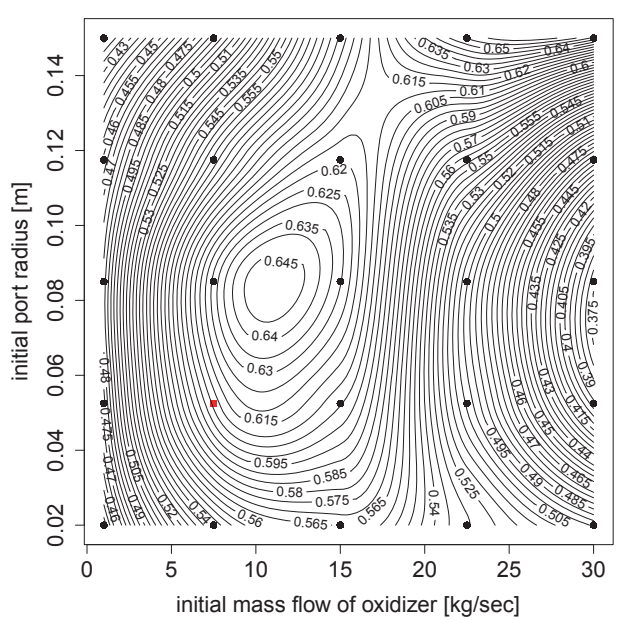

Fig. 5. Aggregated acceptability in flow-radius after calculation \#1.

The results of the aggregated acceptability of all individuals at each calculation time are shown in the boxplots of Fig. 14. The median aggregated acceptability tended to increase from the 0 th to the 4th round of calculations. Among all individuals and all calculations, the individual with the highest aggregated acceptability was in the fourth round of calculations.

\section{DISCUSSION}

Firstly, we discuss on how this approach can narrow the range of design variables (Fig. 4). Fuel length was narrowed the most largely among six design variables; its range became $[2.2,2.6]$ at the forth calculation from $[1,10]$ at the initial setting. Initial mass flow of oxidizer was the second largely 


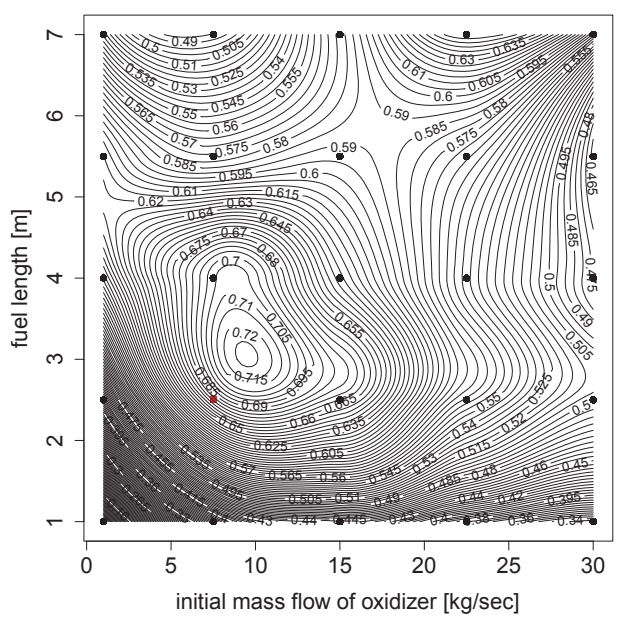

Fig. 6. Aggregated acceptability in flow-length after calculation \#1.

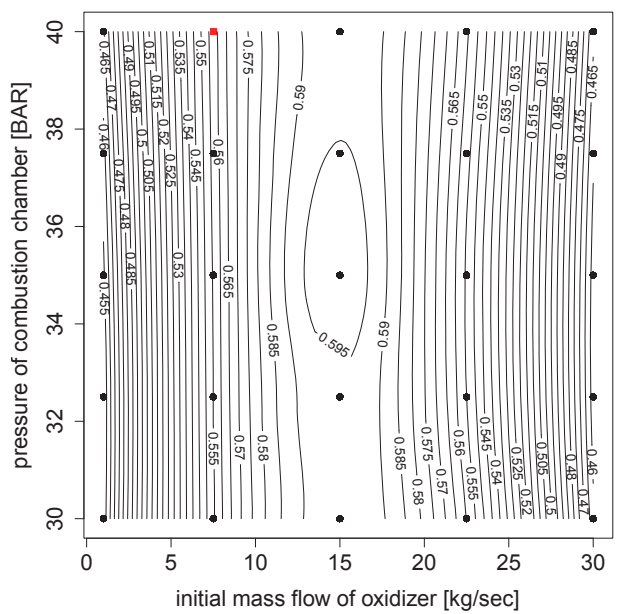

Fig. 7. Aggregated acceptability in flow-pressure after calculation \#1.

narrowed; its range became $[4,8]$ at the forth calculation from $[1,30]$ at the initial setting. The reason of their effectiveness is that these two design variables had clear peaks indicated by the contours of the aggregated acceptability, as shown in Fig.s 6, 10-12, and the position of the highest points (red dots) also located near the peaks. On the other hand, it was hard to narrow the range of combustion time. The ridge of the contour lines in Fig. 8 shows a gentle slope, which makes difficult to narrow down its range by observing the contours of the other design variables visually except Fig. 8. Since the best individuals were always at the minimum value of combustion time in the first and second calculations, we shifted its range down at the third calculation manually, which is the advantage of our proposed method to embed our visual assist in search.

All contour lines in the space of the two design variables at the fourth calculation disappeared (Fig. 13). Since the aggregated acceptability variance of individuals became small at

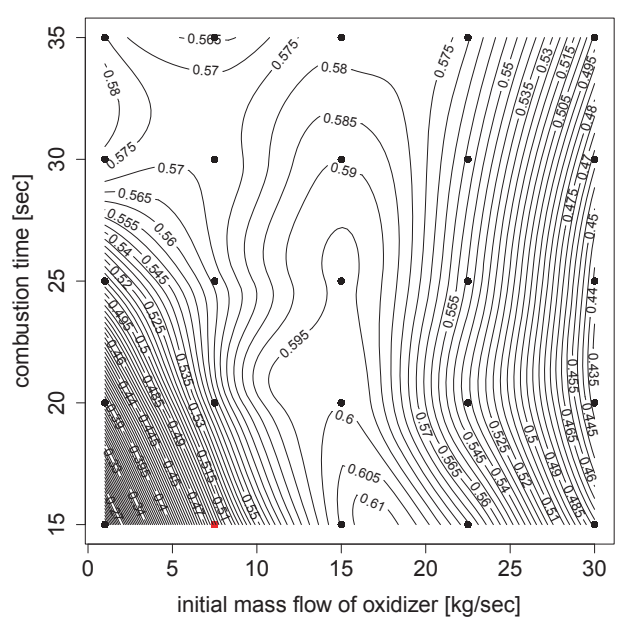

Fig. 8. Aggregated acceptability in flow-time after calculation \#1.

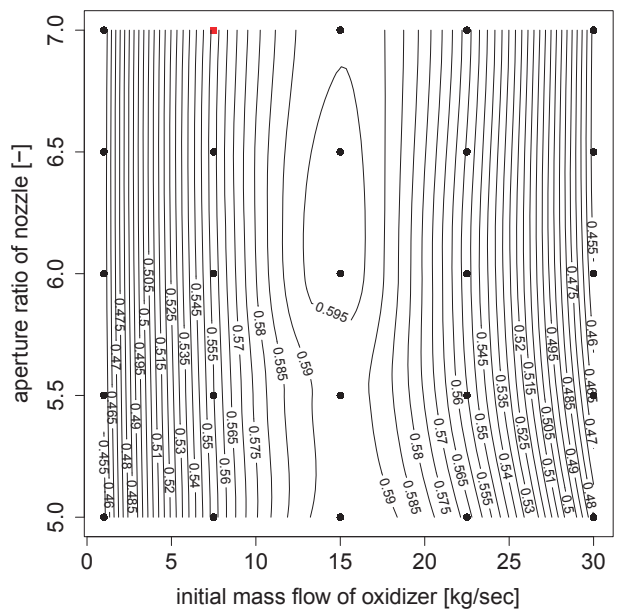

Fig. 9. Aggregated acceptability in flow-nozzle after calculation \#1.

the fourth calculation time as shown in Fig. 14, the aggregated acceptability landscape around the space shown in Fig. 13 may be so flat that contour lines become so sparse. In order to confirm this, we can try to make a plot with the top-ranked individuals in Pareto.

The biggest aggregated acceptability value till the fourth calculation was found at the third calculation time instead of the fourth one (Fig. 14). We think that it shows a convergence trend of solution individuals. The median aggregated acceptability value of all individuals increased substantially from the third calculation time to the fourth one, and there were no more individuals with zero values at the fourth time, which is a good cue for us to narrow search area in the design space.

Comparison of the conventional method [14] and our proposed method is shown in TABLE I. They are the design variable values, the objective values, and the acceptability values of the best individual, i.e. the individual with the 


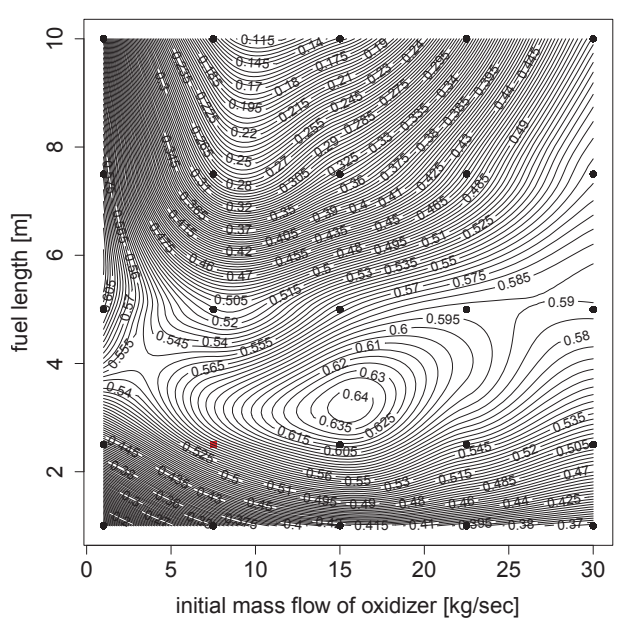

Fig. 10. Aggregated acceptability in flow-length after calculation \#0.

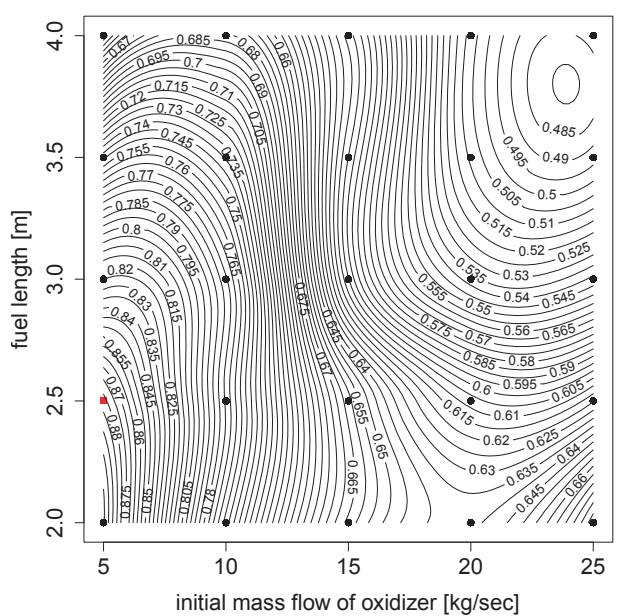

Fig. 11. Aggregated acceptability in flow-length after calculation \#2.

highest aggregated acceptability value. The best individual of the conventional method was obtained by MOGA using 64 population size in 32 generations. We calculated the acceptability values and the aggregated acceptability value of two methods in the table using the same acceptability functions. Although the conventional method and our proposed method performed better for the acceptability of $H_{\max }$ and $W_{\text {gross }}$, respectively, our proposal method performed better for the final index, aggregated acceptability.

The advantage of the proposed method is to be able to handle considerably larger number of individuals. On the other hand, the conventional method used efficient MOGA for optimization, while the proposal method used inefficient manual narrowing of a design space this time. It is necessary to make comparisons under the same conditions in the future,.

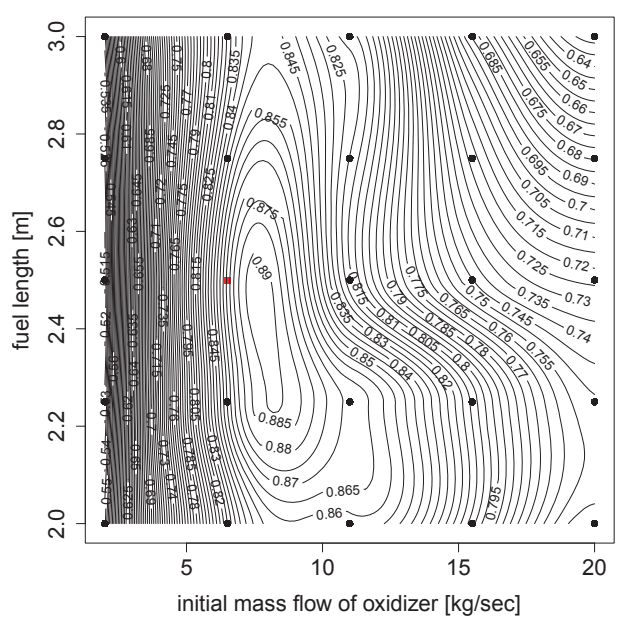

Fig. 12. Aaggregated acceptability in flow-length after calculation \#3.

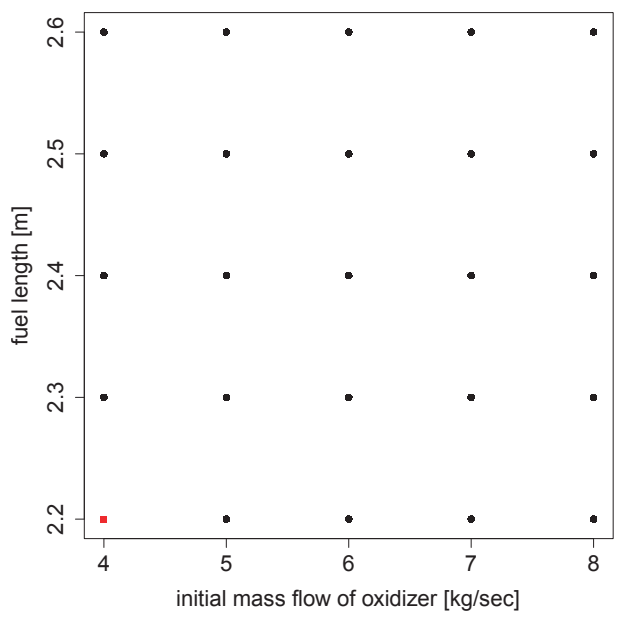

Fig. 13. Aggregated acceptability in flow-length after calculation \#4.

\section{CONCLUSION}

We evaluated our proposed method for multi-objective optimization tasks. The feature of this method is to handle the aggregated acceptability of individuals and narrowed down the range of design variables according to calculations. Since the aggregated acceptability landscape are formed on both an objective space and a design variable space, it becomes a bridge to connect these spaces and lets decision makers, such as design engineers, directly check, examine, and handle the design space of multi-objective optimization tasks. This is the biggest feature and effectiveness of the acceptability concept.

As a result, we are able to find a solution that was superior to the previous study in terms of integration acceptability and better for a single objective. However, the large number of individuals calculated in this study was very advantageous over the conditions of the previous study. In the future, we would like to conduct experiments under the same conditions. 


\section{REFERENCES}

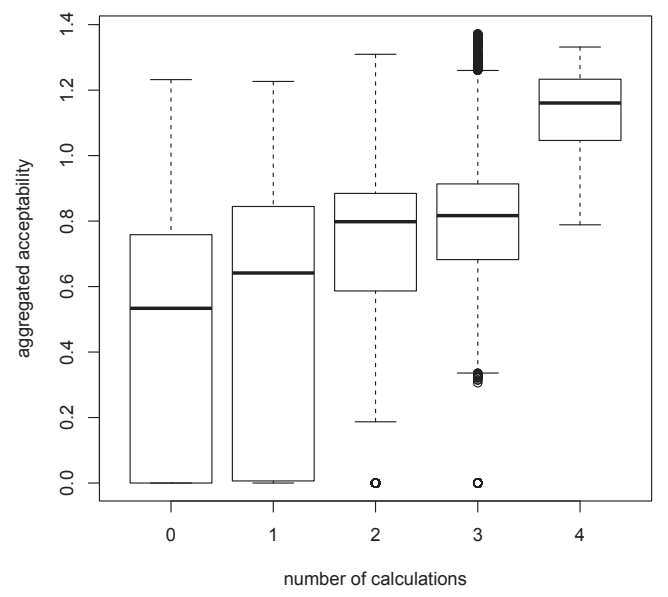

Fig. 14. Aggregated acceptability of all individuals per calculation

TABLE I

COMPARISON OF THE BEST INDIVIDUALS IN CONVENTIONAL AND PROPOSAL METHODS.

\begin{tabular}{|c|c|c|c|}
\hline & & conventional & proposal \\
\hline \multirow{6}{*}{ 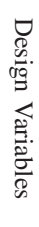 } & initial port radius $[\mathrm{m}]$ & 0.04 & 0.04 \\
\hline & fuel length $[\mathrm{m}]$ & 1.6 & 2.5 \\
\hline & pressure of combustion chamber [BAR] & 39 & 40 \\
\hline & combustion time $[\mathrm{sec}]$ & 26 & 10 \\
\hline & initial mass flow of oxidizer $[\mathrm{kg} / \mathrm{sec}]$ & 3.3 & 6.5 \\
\hline & aperture ratio of nozzle [-] & 6.437 & 6.625 \\
\hline \multirow{2}{*}{$\stackrel{0}{0}$} & $W_{\text {gross }}($ minimize $)[\mathrm{kg}]$ & 269.229 & 217.418 \\
\hline & $H_{\max }(\operatorname{maximize})[\mathrm{km}]$ & 119.562 & 118.587 \\
\hline \multirow{3}{*}{$\begin{array}{l}\overrightarrow{8} \\
\stackrel{8}{8} \\
\stackrel{8}{8}\end{array}$} & $W_{\text {gross }}$ (acceptability) & 0.592 & 0.747 \\
\hline & $H_{\max }$ (acceptability) & 0.630 & 0.623 \\
\hline & aggregated acceptability & 1.222 & 1.371 \\
\hline
\end{tabular}

Although a concrete task in this paper was a two-objective optimization task, we believe that our proposed method using acceptability concept has greater advantage for many-objective optimization.

Decision maker manually narrowed a search area in a design space in our experiment this time. However, we believe that proposed acceptability functions can be easily included in ECs. In the future, we plan to introduce acceptability into the EC algorithm for optimization.

\section{ACKNOWLEDGMENT}

We used the Hybrid Rocket Engine Conceptual Design Evaluation Tool opened by Prof. Akira Oyama at the Japan Aerospace Exploration Agency. We received advice from Prof. Masahiro Kanazaki of Tokyo Metropolitan University and Prof. Akira Oyama on the development of acceptance functions. We hereby express our gratitude.

This work was supported by JSPS KAKENHI Grant Number $18 \mathrm{~K} 11470$ and $20 \mathrm{~K} 12524$.
[1] K. Chiba, H. Yoda, M. Kanazaki and T. Shimada, "Extinction-reignition superiority in a single-stage sounding hybrid rocket," Aerospace Science and Technology, vol.58, no.2016, pp.437-444, 2016.

[2] Kalyanmoy Deb, Multi-objective optimization using evolutionary algorithms, John Wiley \& Sons, Inc., 2001.

[3] K. Deb, A. Pratap, S. Agarwal, and T. Meyarivan, "A fast and elitist multiobjective genetic algorithm: NSGA-II," IEEE Trans. on Evolutionary Computation, vol.6, no.2, pp.182-197, 2002.

[4] K. Deb and J. Sundar, "Reference point based multi-objective optimization using evolutionary algorithms," Genetic and Evolutionary Computation Conference (GECCO2006), pp.635-642, 2006.

[5] K. Deb and H. Jain, "An evolutionary many-objective optimization algorithm using reference point-based non-dominated sorting approach, Part I: solving problems with box constraints," IEEE Trans. on Evolutionary Computation, vol.18, no.4, pp.577-601, 2014.

[6] C. M. Fonseca and P. J. Fleming, "Genetic algorithms for multiobjective optimization: Formulation, discussion and generalization," 5th Int. Conf. on Genetic Algorithms (ICGA'93), pp.416-423, 1993.

[7] M. Gong, F. Liu, W. Zhang, L. Jiao and Q. Zhang, "Interactive MOEA/D for multi-objective decision making," 13th annual conf. on Genetic and evolutionary computation (GECCO2011), Dublin, Ireland, pp.721-728, 2011.

[8] T. Hiroyasu, H. Ishida, M. Miki, H. Yokouchi, "Difficulties of evolutionary many-objective optimization," The science and engineering review of Doshisha University, vol.50, no.1, pp.24-33, 2009, (in Japanese).

[9] M. Inoue, M. Takahashi, Y. Pei and H. Takagi, "Acceptability-based Many-objective Search for Selecting Rental Apartments," 11th Japanese Society for Evolutionary Computation Meeting, pp.183-189, 2016, (in Japanese).

[10] M. Inoue, Y. Pei and H. Takagi, "Design Acceptability for manyobjective optimization - Silent supersonic technology demonstrator as a Task -," 12th Japanese Society for Evolutionary Computation Meeting, pp.81-88, 2017, (in Japanese).

[11] H. Ishibuchi, N. Akedo, H. Ohyanagi and Y. Nojima, "Behavior of EMO algorithms on many-objective optimization problems with correlated objectives," IEEE Congress on Evolutionary Computation (CEC2011), pp.1465-1472, 2011

[12] R. Keeney and H. Raiffa, Decisions with multiple objectives: Preferences and value trade-offs, Cambridge University Press; Revised. 1993.

[13] T. Kishigami and T. Yoshikawa, "A study on search to user's preference direction based on reference lines," Trans. of the Japanese Society for Evolutionary Computation, vol. 6, no. 1, pp.31-41, 2015, (in Japanese).

[14] Y. Kosugi, A. Oyama, K. Fujii and M. Kanazaki, "Conceptual design optimization of hybrid rocket engine," Proceedings of Space Transportation Symposium, 2010, (in Japanese).

[15] S. Motomura and H. Takagi, "Study on retrieval of rental apartments database using acceptability functions," 34th Fuzzy System Symposium, Nagoya, Japan, pp.775-780 (September 3-5, 2018). (in Japanese).

[16] Akira Oyama, "Optimization problems in space science, conceptual design optimization of hybrid rocket engine", https://ladse.eng.isas.jaxa. jp/benchmark \_space/, 2020, (in Japanese).

[17] H. Takagi, M. Inoue and Y. Pei, "Introduction of acceptability to multi-objective optimization," 9th Japanese Society for Evolutionary Computation Meeting, pp.18-23, 2015, (in Japanese).

[18] Q. Zhang and H. Li, "MOEA/D: A multiobjective evolutionary algorithm based on decomposition," IEEE Trans. on Evolutionary Computation, vol.11, no.6, pp.712-731, 2007. 